\title{
Prédiction directe de la qualité des ensilages préfanés par la spectrométrie dans le proche infrarouge
}

\author{
G Sinnaeve, P Dardenne, P Lecomte, R Agneessens, Y Seutin \\ Station de Haute Belgique (CRA-Gx), 6800 Libramont, Belgique
}

L'analyse des fourrages en spectrométrie dans le proche infra rouge s'applique généralement à des produits séchés et broyés. Le but de cette étude est le développement de modèles prédictifs permettant d'estimer la composition et l'état de conservation d'ensilages préfanés, la saisie des spectres s'opérant directement sur le fourrage en l'état, sans préparation préalable.

Les échantillons (150) ont été prélevés dans les stocks de balles rondes enrubannées de 4 exploitations du plateau Ardennais. Les fourrages récoltés à différentes époques et stades étaient représentatifs d'une importante variabilité quant à la composition et aux paramètres fermentaires. Les spectres ont été acquis à la fois sur le matériel frais et sur les poudres après séchage et broyage au moulin Cyclotec $(1,0 \mathrm{~mm})$. Les échantillons ont tous été analysés sur frais pour les critères : matière sèche réelle ( $\mathrm{MSR}, \%$ ), $\mathrm{pH}$ et azote ammoniacal $\left(\mathrm{N}-\mathrm{NH}_{3}, \mathrm{mg} \mathrm{N} / 100 \mathrm{~g}\right.$ de produit frais) selon les méthodes de référence. Sur base de procédures de sélection spectrale, 15 échantillons ont été retenus pour être analysés par méthode de référence quant à leur teneur en protéines (MPT, \% MS), cellulose brute
(CEL, \% MS), cendres totales (CT, \% MS) et digestibilité à la pepsine cellulase (CasedMO, $\% \mathrm{MO})$. Ces déterminations effectuées sur les poudres (broyage cyclotec) ont permis de conforter les modèles prédictifs établis précédemment sur échantillons broyés pour les critères de composition et de prédire l'ensemble des échantillons. Les valeurs ainsi prédites ont été reportées sur les spectres des ensilages frais. L'établissement de calibrages sur les données spectrales des 150 échantillons mesurés en frais conduit aux erreurs standards (SEC) et corrélations $\left(R^{2}\right)$ reprises au tableau.

Compte tenu de ces résultats, on peut considérer que les calibrages établis sur, d'une part, les spectres des échantillons préfanés frais et d'autre part, sur les valeurs des échantillons séchés et broyés aboutissent à des modèles prédictifs intéressants. Les précisions sont du même ordre de grandeur que celles obtenues sur produits séchés et broyés mais le mode d'acquisition des spectres permet une évaluation directe de la matière sèche et de la qualité des ensilages préfanés en balles rondes et ce dès l'ouverture des balles.

\begin{tabular}{lcccc}
\hline & Unités & Min - Max & SEC & $R^{2}$ \\
\hline MPT & $\%$ MS & $8-23$ & 1,08 & 0,91 \\
CEL & $\%$ MS & $21-36$ & 1,00 & 0,91 \\
CT & $\% \mathrm{MS}$ & $5-18$ & 0,87 & 0,87 \\
CASEDMO & $\% \mathrm{MO}$ & $51-86$ & 2,35 & 0,91 \\
MSR & $\%$ & $22-85$ & 2,79 & 0,95 \\
pH & - & $3,1-6,5$ & 0,35 & 0,67 \\
$\mathrm{~N}-\mathrm{NH}_{3}$ & mg N/100 g de frais & $0-118$ & 11,23 & 0,77
\end{tabular}

